\title{
SISTEMA DE INDICADORES PARA MEDIR EL COMPORTAMIENTO DEL CONSUMIDOR DEL DESTINO TURístico SuCre SAN Vicente
}

\author{
Frank Ángel Lemoine Quintero ${ }^{1}$, Lilia Moncerrate Villacis \\ Zambrano $^{2}$, Norma Rafaela Hernández Rodríguez ${ }^{3}$, Daniela \\ Liceth Panta Ronquillo ${ }^{4}$ y Graciela Castellano Pallerols 5
}

Recibido: 2/2/2020 • Aceptado: 9/15/2020

\begin{abstract}
Cómo citar: Lemoine Quintero, F. Ángel, Villacis Zambrano, L. M., Hernández Rodríguez, N. R., Panta Ronquillo, D. L., \& Castellano Pallerols, G. (2021). Sistema de indicadores para medir el comportamiento del consumidor del destino turístico Sucre San Vicente. Ciencia, Economía y Negocios, 5(2), 61-84. Doi: https:// doi.org/10.22206/ceyn.2021.v5i2.pp61-84
\end{abstract}

\section{Resumen}

El objetivo fue identificar los indicadores de comportamiento del consumidor que permita gestionar la sostenibilidad de la actividad turística. Se diseñó un cuestionario de 12 preguntas y de 5 escala para medir niveles de satisfacción de los consumidores en el destino. De los resultados de la encuesta los más representativos correspondieron a la variedad de las ofertas turísticas que brinda hoteles y servicios del restaurante donde un 42,1\% de los encuestados responden que es lo esperado y concerniente a la seguridad turística el 43,2\% de los encuestados opinaron es igual a lo esperado denotando un destino seguro. Se conformó un sistema de indicadores a través de la revisión de literatura y se puso a consideración de los expertos para su estimación a través de la aplicación del método Delphi, donde el coeficiente de concordancia resultó ser de 0,85 valorado de muy bueno. La metodología utilizada evidenció los diez indicadores que contribuirán a un comportamiento del consumidor acorde a las exigencias del destino turistico.

Palabras clave: Método DELPHI, indicadores de turismo, comportamiento del consumidor, sostenibilidad.

Código JEL: D12, D16, D22

\footnotetext{
${ }^{1}$ Universidad Laica Eloy Alfaro de Manabí, Manabí, Ecuador. ORCID: 001-8885-8498 Correo-e: flemoine1964@gmail.com

${ }^{2}$ Universidad Laica Eloy Alfaro de Manabí. ORCID: 0002-2888-6363 Correo-e: liviza@hotmail.com

${ }^{3}$ Universidad de Oriente, facultad de Ciencias Económicas y Empresariales, Santiago de Cuba, Cuba. ORCID: 0002-2086-2236, Correo-e: norma@uo.edu.cu

${ }^{4}$ Universidad Laica Eloy Alfaro de Manabí. Correo-e: danliceth-p29@hotmail.com

${ }^{5}$ Universidad de Oriente, facultad de Ciencias Económicas y Empresariales, Santiago de Cuba, Cuba.
} 


\title{
Indicator Systems for Measuring Consumer BEHAVIOR: APPLICATIONS TO THE TOURISM SECTOR
}

\author{
Frank Ángel Lemoine Quintero, Lilia Moncerrate Villacis \\ Zambrano, Norma Rafaela Hernández Rodríguez, Daniela \\ Liceth Panta Ronquillo and Graciela Castellano Pallerols
}

Received: 2/2/2020 Approved: 9/15/2020

\begin{abstract}
Our objective is to identify indicators to measure consumer behavior that allows assessing tourist activity sustainability. We designed a survey with 12 questions and five scale answers to capture consumer satisfaction at the touristic destination. Then, we use the Delphi method combined with expert's opinions to identify the leading indicators that will allow us to assess consumer's behavior in ht region. We identify a total of 10 indicators with a level of concordance of 0.85 points.
\end{abstract}

Keywords: leading indicators, tourism, sustainability, Delphi method.

JEL Codes: D12, D16, D22 


\section{Introducción}

El servicio de alojamiento en el Ecuador forma parte de las microempresas en el país, contribuyendo al desarrollo socioeconómico de las comunidades y localidades, además de acelerar la generación de empleos e incrementar el ingreso familiar por la particularidad de que estas constituyen emprendimientos familiares. Tiene la ventaja de aprovechar los recursos internos que existen en las áreas urbanas y rurales como oportunidad para posicionarse en el mercado tanto local, regional, nacional e internacional.

Es importante argumentar que el Consejo Empresarial para el Desarrollo Sostenible del Ecuador (CEMDES) estableció el Plan Nacional de Desarrollo y su continuidad con el Plan Nacional para el Buen Vivir, como carta de navegación del país. Enfatizó que dentro de los objetivos de desarrollo del plan se incluye la dinámica privada, a través de sus correspondientes estrategias en los diferentes sectores, que a su vez permite impulsar actividades sustentables a nivel de las empresas de servicios hoteleros.

Cabe fomentar que, según Vargas, Vaca \& Garcia (2014) la política del turismo sostenible es de un enfoque positivo, encaminada a mantener productos y servicios turísticos de calidad, acompañada de instrumentos de gestión adecuados para contribuir a la conservación del medio ambiente y al bienestar socio-económico; este no es un enfoque temporal. Las zonas naturales y sus entornos obtienen cada vez más valor, y las actividades que se desarrollan en las mismas se comprometen a su protección y conservación a largo plazo cuando operan bajo una filosofía de sostenibilidad turística.

En la actualidad el turismo es reconocido como prioridad nacional con el establecimiento de las políticas de Estado para el sector en la Estrategia de Desarrollo del país, por lo cual el Gobierno Nacional presentó la Política Nacional de Turismo, que convertirá al país en potencia turística. (Ministerio de Turismo del Ecuador, 2017). Toda actividad turística solidaria siempre que admita la participación activa de la comunidad desde una perspectiva intercultural, manejo adecuado del patrimonio natural y valoración del patrimonio cultural, basados en un principio de equidad en la distribución de los beneficios locales en bien común de las comunidades existente en el país, por lo que se trabajan principalmente 
4 ejes: revitalización cultural, socio-organización, economía solidaria y defensa territorial (PEGEASOSTENIBLE, 2012).

En el 2016 el país presento aproximadamente 4.500 establecimientos de alojamiento turístico, de los cuales solo 27 eran de lujo, que significa menos del $0,5 \%$ con una tarifa promedio de $\$ 110$, cuando esto en los países de la región está sobre $\$ 170$. En el caso de los hoteles de 4 estrellas no superaron el $6 \%$ con una tarifa promedio de $\$ 70$, sin embargo, la mayoría se encontraron en el rango de tarifas inferiores a $\$ 45$. Con estas cifras fue muy difícil tener recursos para hacer inversión en mejoramiento (Sánchez, 2015).

OMT (2018) informó que el turismo en Ecuador reflejó un $14 \%$ crecimiento en el 2017 con respecto al ańo anterior, resaltando que el $44,2 \%$ de turistas extranjeros corresponde a los denominados mercados naturales registrando un crecimiento de $0,5 \%$. Los mercados objetivos figuran en el país el 20,9\% donde han registrado un decrecimiento de $17,5 \%$. A pesar de lo anteriormente indicado ha existido un $13 \%$ de aumento en los ingresos por concepto de turistas días y por alojamiento con representatividad nacional, personificado el turismo el 1,6\% del PIB a nivel de mercado ecuatoriano. El 2018 muestra mejora en los indicadores ingresos (12.91\%), entrada de turismo (51.57\%) y en lo que representa en el PIB $(1,7 \%)$, aunque han sido distintivos aún no cumplen con el plan estratégico para de turismo para algunos destinos del país.

El destino cuenta con un total de 123 infraestructura hoteleras donde el $57.72 \%$ están ubicados en el cantón San Vicente y el $41.46 \%$ en el cantón Sucre identificados estas infraestructuras como hotel, hostales, pensión, cabañas y campamentos, conformado por 5134 plazas y 1678 habitaciones a nivel de destino.

Los cantones Sucre y San Vicente ubicado en la litoral costa considerada como un destino de balnearios de sol y playa, poseen además atractivos turísticos categorizados y jerarquizados por el Ministerio de Turismo del Ecuador, los cuales fortalecen a las empresas hoteleras y gastronómicas en todos los servicios que ofrecen, convirtiéndolo en un destino para los turistas. Este desarrollo turístico a su vez es un pilar fundamental para el crecimiento económico del destino, tanto en la rama turística como hotelera, en dependencia de la gestión comercial del destino. 
Estudios revelan que el comportamiento del consumidor se ha convertido en un componente integral de la planeación estratégica del marketing, en el que la creencia de la ética y la responsabilidad social también debe ser parte fundamental de toda decisión comercial. El comportamiento del consumidor se basa en conceptos y teorías respecto del ser humano, desarrolladas por diversas disciplinas, como la psicología, la sociología, la psicología social, la antropología cultural y la economía (Escalante, 2016).

El Campus de Bahía de Caráquez de la Universidad Laica Eloy Alfaro de Manabí en su papel protagónico en las comunidades ha desarrollado investigaciones con vista a fortalecer la actividad turística. Partiendo de las leyes y políticas existentes en el país tales como: Ley del Turismo, PLAN DE TOURS 2020, Plan Integral de Marketing Turístico para el Turismo Interno de Ecuador (PIMTE 2014), código orgánico de la producción de comercio e inversiones, normas del sector de alojamiento y Plan Nacional del Buen Vivir ha considerado la revisión, análisis y estudio de estas para valorar su cumplimiento y sus aportes para el desarrollo turísticos de los cantones Sucre y San Vicente.

El Observatorio Turístico de la Extensión Universitaria luego de realizar un diagnóstico a nivel de destino ha observado el bajo índice de ingreso por turista en el sector y deficiente índice de repitencia de turista al destino, lo que ha conllevado a un desequilibrio en actividades comerciales y de servicios que provocan bajo niveles de eficiencia en el turismo. El objetivo de esta investigación es definir indicadores de comportamiento del consumidor del destino turístico Sucre-San Vicente que permita gestionar el fortalecimiento de la actividad turística, aumentar el nivel de repitencia y fidelización de los consumidores en el destino.

Estudios relacionados a la temática de turismo han demostrado que el método Delphi aporta significativamente a resultados investigativos efectivos y diversos, a procedimientos que acometen planteamientos muy variados entre los que destacan aquellos que abordan el análisis de destinos turísticos y sus necesidades en cuanto a planificación turística. Fernández (2012) aborda referente a la oportunidad y conveniencia de aplicar la prospectiva al sector turismo mediante la planificación turística con la finalidad de limitar la incertidumbre que acompaña sus acciones e inversiones a largo plazo, por otro lado Picazo et al (2013) realizan un estudio de los 
aportes de la investigación turística que los investigadores e instituciones realizan en el ámbito turístico a partir del método Delphi.

Resulta importante referir que Gallardo ( 2013) aplica el método para evaluar el potencial turístico en las playas del departamento del Atlántico, desde la perspectiva ambiental, mientras que Solsona (2014) desde el análisis prospectivo del turismo rural utilizan los parámetros de ofertas y demanda, gestión turística, infraestructura y servicios públicos entre otros para determinar los factores limitantes y dificultades para el desarrollo turístico rural, aunque Torres (2016) determina que los principales factores que afectan la evaluación de la sostenibilidad del desarrollo turístico en Cuba a partir de resultados del método Delphi los más relevantes fueron la inconsistencia en las metodologías de evaluación y en los datos, así como la no implicación de todas las partes interesadas que ha traído consigo delimitaciones de los destinos turísticos,siendo cada criterio importantes para lograr destino sostenibles.

Morales et al (2018) a través de un cuestionario evalúa el nivel de satisfacción e intención de repetir la visita turística a las playas de Ecuador a través del método planteando nueve atributos ( variedad de productos turísticos, seguridad, precios económicos, calidad de los productos turísticos, infraestructura hotelera, cercanía, publicidad, canales de distribución y clima) seleccionados para el presente estudio donde los resultados confirman que los atributos variedad de productos, precios, canales de distribución y clima son predictores en la intención de repetir la visita al lugar de destino, lo que se pueden consideran estos representativos para medir los indicadores de comportamiento del consumidor turístico en el presente estudio.

Otros análisis y aplicaciones del método Delphi están relacionadas con: determinar el potencial del turismo rural en oasis sudcalifornianos de México (Ruíz, 2016); determinar el impacto del turismo en destinos clasificados como patrimonios de la humanidad (Pulido \& Ruíz, 2017; aplicar el diseño de un modelo de financiación de transporte urbano como la mejor opción metodológica (Flores et al 2019) ; definir un procedimiento para evaluar la localización de los servicios complementarios en la red extra hotelera en destinos turísticos a partir de la determinación de un índice de acercamiento al cliente y su relación con el atractivo (Gómez et al 2020); comprobar resultados de los once indicadores establecidos en 
el instrumento de validación del modelo para establecer la gestión sostenible para el desarrollo turístico en Ecuador (Garcia et al 2020)

Por todo lo antes expuestos y por el estudio de realizado por Cruz \& Rúa (2018) referente a la exploración en Google Académico, fechada el 9 de marzo de 2017, refleja la marcada preferencia por los términos Delphi method (51200), Delphi study (44500), y Delphi technique (39500). Estos valores son superiores a los observados para Delphi methodology (5060), Delphi research (3540), y Delphi design (609), por lo que denota la utilización del método en todo el ámbito investigativo y científico por sus especificaciones, cualidades y maniobrabilidad para la obtención de resultados efectivos.

\section{Sistema de indicadores}

A partir de revisiones bibliográficas que aporten al estudio de indicadores para medir el comportamiento del consumidor en el destino objeto estudio se consideraron los siguientes criterios de autores relacionado a la temática:

Criterios abordados por Solomon (2008) relaciona que los mercadólogos necesitan entender los deseos y las necesidades de distintos segmentos de consumidores, considerando dentro de los indicadores demográficos los aspectos objetivos de una población, como la edad y el sexo y los dentro de los psicográficos las características psicológicas y del estilo de vida, además de considerar a los indicadores gráficos contra verbales para destino turísticos emergentes.

Por lo que es importante considerar el planteamiento de Narváez \& Fernández (2010) referente al turismo desde la perspectiva de la demanda relacionados los indicadores socio-económicos y demográficos, lugar de procedencia y nivel de satisfacción a partir de la segmentación del mercado y de las especificaciones del destino.

Otros criterios referente a la temática de los autores Conde et al 2013 indican que los factores que influyen en el comportamiento del consumidor turista y la gestión de la mercadotecnia de destino turístico a partir de los indicadores de desempeño y del destino es agrupándoles en: I-Perfil del visitante, motivo del viaje, duración, grupo de viaje, transporte, hospedaje, II- Fuentes de información sobre el destino, III- Situación económica, ingreso disponible para viajar, percepción de los precios de servicios 
y VI- Evaluación de atributos del destino, intenciones conductuales, para obtener resultados favorables en un destino turístico.

Un estudio relacionado con los perfiles turísticos en función de las motivaciones para viajar ha determinado ochos indicadores (Explorar, Escape, Prestigio, Relajación, Nostalgia, Relación Personas, Interacción Social, Cultura y Novedad) a partir de la obtención de clusters o perfiles turísticos en función de las motivaciones turísticas, siguiendo el modelo de Crompton donde a partir de un análisis de conglomerados obtuvieron cuatro perfiles turísticos: racionales, antropológicos, emocionales y hedonistas favorables propio para el destino de la Región de Murcia y las provincias de Alicante y Almería. Beltrán \& Parra (2017)

Carvajal et al 2017 en sus estudios de gestión por procesos facilitan los indicadores adecuados para obtener la clave del éxito de una gestión por procesos, estos son: los indicadores de eficacia y los de eficiencia, considerando que a partir de estos indicadores se logra gestionar la organización, además de identificar y clasificar los indicadores atendiendo a su naturaleza, alcance y determinar la orientación de la gestión de la organización.

Por otro lado, Carbache et al 2018 enfatiza referente a los indicadores motivaciones, valoración y satisfacción del turista en un destino de Sol y playa de Ecuador, considerando que estos indicadores forman parte de la lealtad de los visitantes cuya sinergia formarían una estructura sólida que contribuyen de manera significativa a la calidad de los servicios y por consiguiente a la lealtad del destino.

Lemoine et al 2020 desde la fundamentación de su estudio referente al comportamiento del consumidor en la gestión comercial de destinos turísticos Sucre-San Vicente realizan una valoración referente a los criterios de indicadores de Hawkins, Best, y Coney (2004), a su vez, pertinente a Kotler y Armstrong (2007) en su libro Fundamentos de Marketing donde argumenta sobre los indicadores de satisfacción y puntualiza referente a los índice de importancia relativa de los atributos (IIR), índice de desempeño de los atributos de calidad (IDAC), índice de desempeño de las áreas de atributos de calidad (IDAR), índice satisfacción del cliente con calidad percibida (ISCP), índice de satisfacción con el precio percibido (ISPP) y el índice de satisfacción del cliente (ISC), lo que se consi- 
deran debido a la complejidad del sector turístico en Ecuador, donde se exige que en la evaluación del cliente adopte un enfoque integrado de las dimensiones de la realidad social, no obstante, en la teoría se observa una tendencia a priorizar el enfoque financiero o el enfoque socioeconómico de la teoría neoclásica y no fundamentalmente los aspectos de expectativas y satisfacción de los mismos.

Una vez revisado la conceptualización de sistema de indicadores, se refleja que indicadores son parte de una cadena en una relación causaefecto, orientados a resultados eficaces dependiendo del enfoque que tenga, siempre y cuando se basan en datos históricos y relacionarlo con la actualidad y las posibles evaluaciones que se esperan, es necesario que los indicadores sean un proceso y no complicado. El método de expertos DELPHI determinara cuales son los indicadores que aportan al comportamiento del consumidor del destino turístico Sucre- San Vicente.

El método descriptivo permitirá desde su concepción realizar una descripción del objeto de estudio que permita valorar desde la fundamentación bibliográfica hasta investigaciones referente al estudio determinar los indicadores de comportamiento de los consumidores. La población seleccionada es el destino Sucre-San Vicente, principalmente los balnearios de mayor asistencia de turistas en la temporada de carnaval. La muestra es aleatoria simple, no se estima el número de visitantes para esta fecha. Se utilizó el software estadístico SPSS versión 21 para procesar las encuestas aplicada.

\section{Resultados}

Los servicios hoteleros son fuente generadora de empleo para las personas, están surgen como un intercambio de servicios de calidad, de prestación de servicio y atención al cliente, con la finalidad de que el huésped quede satisfecho con la atención solicitada.

La elaboración de procedimientos con técnicas y métodos matemáticos, permite determinar el nivel de ocupación hotelera, para darle paso al análisis de los factores que provocan una alta o baja demanda económica en los establecimientos hoteleros. Si este porcentaje no es elevado el empresario debe implementar estrategias o técnicas para atraer a más huéspedes a su establecimiento hotelero. 
Se diseñó un cuestionario de 12 preguntas y de 5 escala que permita obtener resultados que permitan la toma de decisiones y estrategias de posicionamiento, además que permita a través de la herramienta estadística, SPSS 21.0 realizar diferentes análisis y determinar la fiabilidad del mismo para comprobar que los huéspedes están satisfechos con el servicio que brinda el sector hotelero del destino. El tamaño de la muestra aleatoria simple fue de 95 turistas que frecuentan en los feriados del carnaval.

Entre los resultados más relevantes esta la respuesta referente a la variedad de las ofertas turísticas que brinda los hoteles para conocer las expectativas de los consumidores de los servicios hoteleros y de restaurantes, cuyos resultados se reflejan en la figura 1.

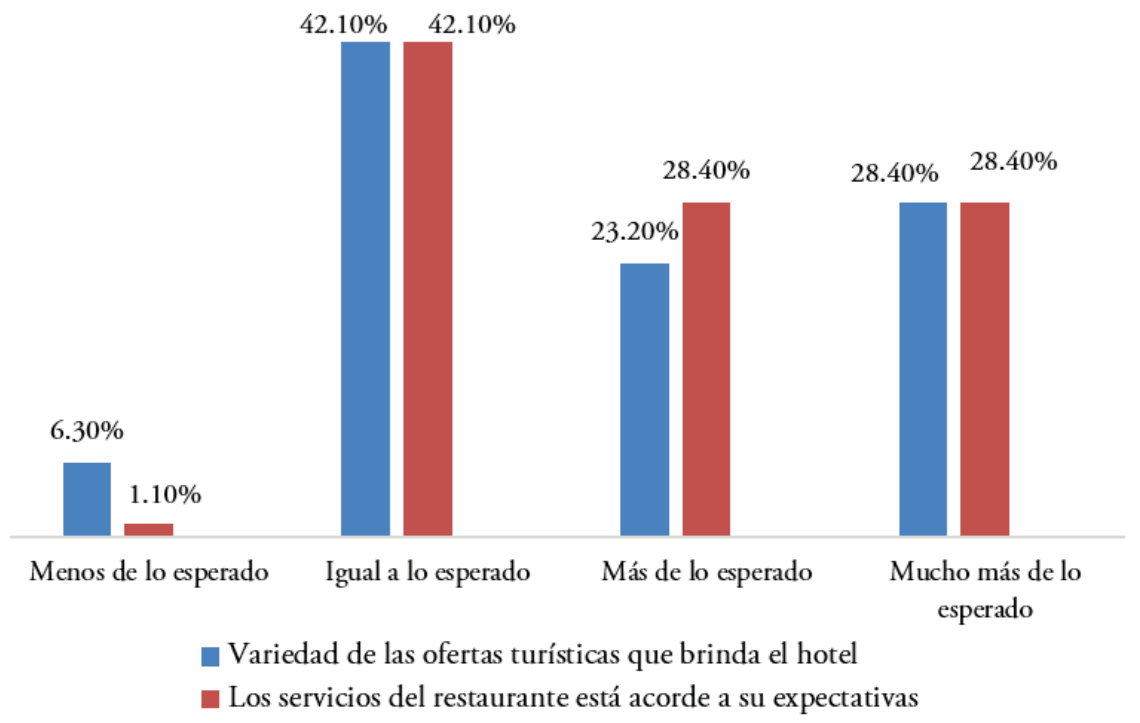

Figura 1: Variedad de las ofertas turísticas

Notas: Encuesta a hoteles y servicios gastronómicos para medir expectativas de los consumidores

En relación a la variedad de las ofertas turísticas que brinda el hotel y a los servicios del restaurante que está acorde a sus expectativas, el 42,1\% reconoce que es lo que ellos esperaban, el 28,4\% sugirió que es mucho más de lo esperado. El 28,4\% que es más de lo esperado y el 1,1\% que es menos de lo esperado, estos dos últimos porcentajes se refieren a la 
pregunta si los servicios del restaurante están acordes a sus expectativas, mientras que varía en; más de lo esperado con el 23,2 \% y el 6,3\% que es menos de lo esperado en el siguiente punto.

Como observamos existe una correlación respecto a las dos interrogantes, ya que se refieren al grado de satisfacción de los huéspedes, por lo cual se caracterizan como aspectos muy importantes en el ámbito de la eficacia y eficiencia en el desarrollo económico de las empresas hoteleras, como se refleja en la figura 2.

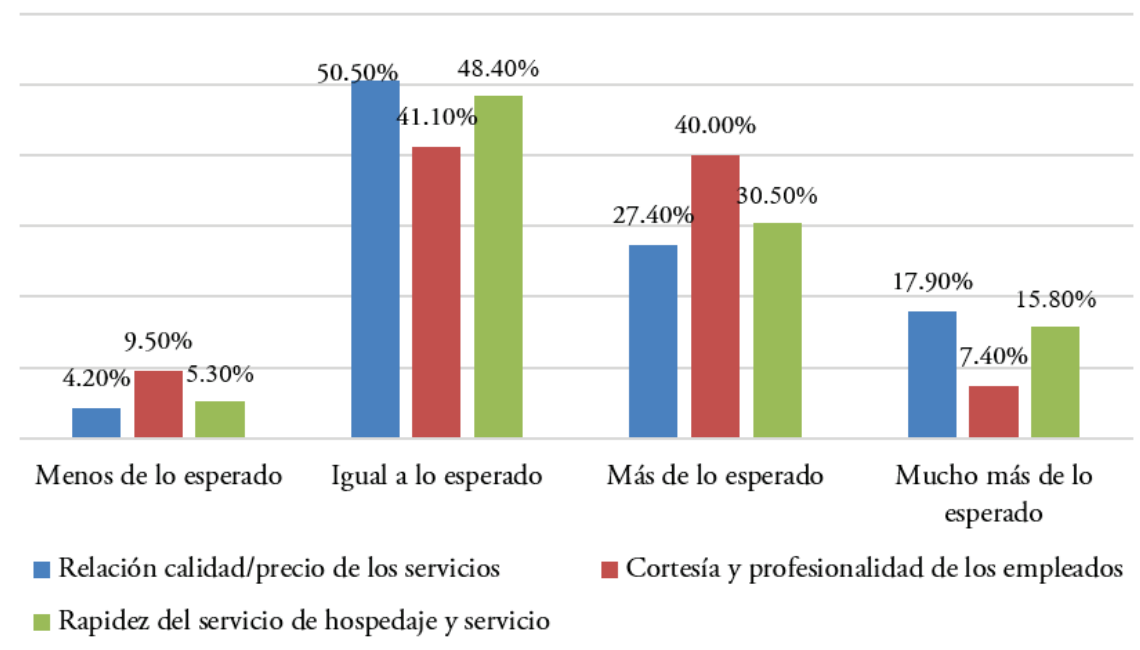

Figura 2: Elementos de eficiencia respecto al servicios

Estas tres variables se relacionan ya que sus niveles en los diferentes aspectos tienen proporciones casi iguales en las mismas dimensiones. En mayor porcentaje los huéspedes manifestaron que es lo que ellos esperaban en cuanto a la relación precio/calidad de los servicios, la cortesía/ profesionalismo de los empleados y la rapidez del servicio de hospedaje y servicios. Seguidamente de la opción más de lo esperado, luego mucho más de lo esperado y por último menos de lo esperado.

Los empleados deben poseer habilidades y conocimientos necesarios para brindar el servicio, de forma correcta y precisa; una sonrisa, un trato amable es la bienvenida al éxito de la empresa de hospedaje. La profesionalidad en el ámbito hotelero implica conocer de manera profunda su 
trabajo y mantener una relación estrecha con el cliente, aconsejándoles o sugiriéndole un servicio, tal como se muestra en la figura 3.

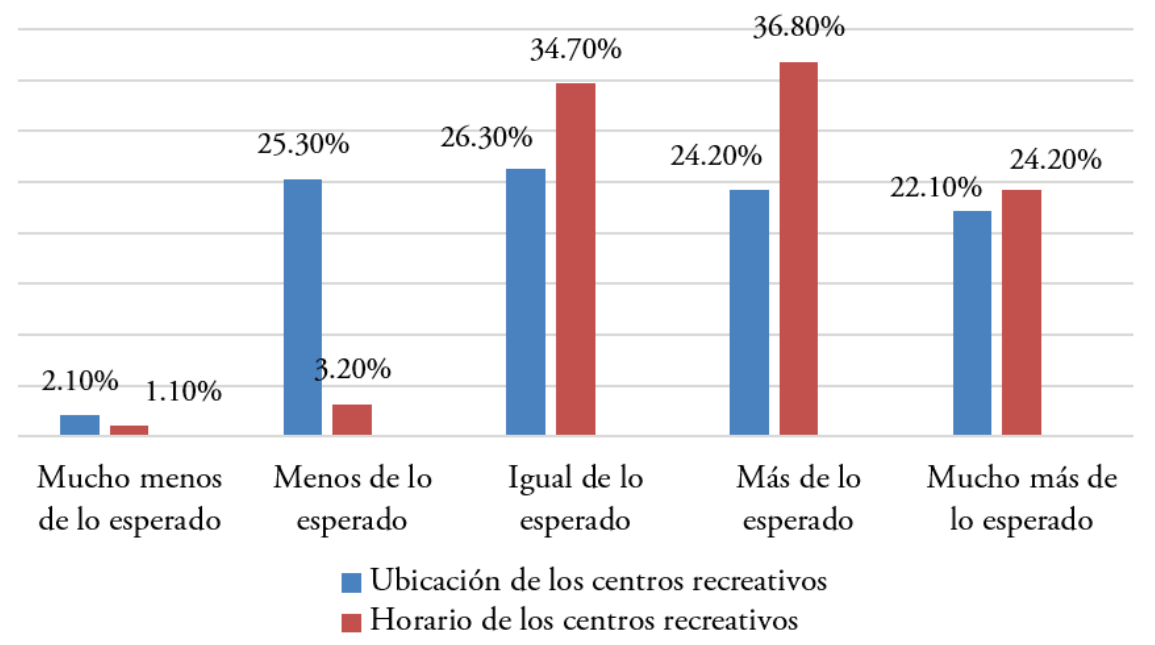

Figura 3: Ubicación y horarios de los centros recreativos

Respecto a la ubicación de los centros recreativos los encuestados concluyeron en mayor porcentaje con $26,3 \%$ que era lo que ellos esperaban, el 25,3\% comentaron que es menos de lo que ellos esperaban, $24,2 \%$ más de lo esperado, $22,1 \%$ mucho más de lo esperado y $2,1 \%$ mucho menos de lo esperado. Así mismo los porcentajes en relación a los horarios tuvieron resultados casi parecidos, el 36,8\% opinaron que es más de lo que ellos esperaban, el 34,7\% dijeron que es igual a lo que ellos esperaban, el 24,2\% que es mucho más de lo que ellos esperaban, el $3,2 \%$ que es menos de lo esperado y $1,1 \%$ que es mucho menos de lo que ellos esperaban.

Esto quiere decir que la localización y el horario de los centros recreativos son factores importantes, para los turistas a la hora de escoger un lugar para pasar las vacaciones o descansar, la mayoría de personas buscan sitios con atractivos turísticos, cerca de una playa o balneario, donde el acceso sea adecuado, la gastronomía sea excelente, o donde se pueda realizar diferentes actividades de recreación según se muestra en los resultados tabla 1. 
Tabla 1. Seguridad turística

\begin{tabular}{lll}
\hline Parámetros & Encuestado & Porcentaje \\
\hline Mucho menos de lo esperado & 1 & $1.1 \%$ \\
\hline Menos de lo esperado & 13 & $13.7 \%$ \\
\hline Igual a lo esperado & 41 & $43.2 \%$ \\
\hline Más de lo esperado & 31 & $32.6 \%$ \\
\hline Mucho más de lo esperado & 9 & $9.5 \%$ \\
\hline Total & 95 & $100 \%$ \\
\hline
\end{tabular}

Fuente: Elaborado con datos propios a través de resultados del SPSS.21.0

En esta tabla podemos observar que el $43,2 \%$ de los encuestados opinaron que la seguridad turística en este hotel es igual a lo esperado, el $32,6 \%$ respondieron que es más de lo esperado, el $13,7 \%$ que es menos de lo esperado, el 9,5\% es mucho más de lo esperado y el 1,1 comentaron que es mucho menos de lo que ellos esperaban. Es decir que la mayoría de turistas que visitan este hotel consideran que este establecimiento hotelero presenta condiciones de seguridades óptimas y por lo tanto aceptables para que los huéspedes puedan estar tranquilos a la hora de hospedarse, cuyos resultados se revelan en la tabla 2.

Tabla 2. Satisfacción de la estancia en el destino sucre-san vicente

\begin{tabular}{lll}
\hline Parámetros & Encuestado & Porcentaje \\
\hline Mucho menos de lo esperado & 1 & $1.1 \%$ \\
\hline Igual a lo esperado & 38 & $40.0 \%$ \\
\hline Más de lo esperado & 33 & $34.7 \%$ \\
\hline Mucho más de lo esperado & 23 & $24.2 \%$ \\
\hline Total & 95 & $100 \%$ \\
\hline
\end{tabular}

Fuente: Elaborado con datos propios a través de resultados del SPSS.21.0 
En este último gráfico observamos que los encuestados se encuentran satisfechos con su estancia en el destino Sucre San Vicente, ya que la mayoría con un $40,0 \%$ dijeron que es igual a lo que ellos esperaban, seguidamente está el 34,7\% de personas que opinaron que es más de lo esperado, el 24,2\% que es mucho más de lo que ellos esperaban y el 1,1\% que es mucho menos de lo esperado.

El sector hotelero del destino a pesar de la eventualidad del terremoto que ocurrió el año 2016 dejando más del 70\% de la infraestructura hotelera afectadas, brinda servicios que satisfacen a los turistas que la visitan, además su entorno sociocultural (atractivos turísticos, las tradiciones, gastronomía, desarrollo social, costumbres, entre otros), son estrategias de eficiencia y eficacia que promueven la demanda turística en este cantón.

La fiabilidad del cuestionario es aceptable porque el valor de la misma estuvo en el rango requerido para definirla como buena según criterios de expertos, tal como se muestra en la tabla 3.

Tabla 3. Resultados de fiabilidad

\begin{tabular}{lll} 
Alfa de Cronbach & $\begin{array}{l}\text { Alfa de Cronbach } \\
\text { basada en los elementos } \\
\text { tipificados }\end{array}$ & No. de elementos \\
\hline, 877 &, 877 & 95 \\
\hline
\end{tabular}

Fuente: Elaborado con datos propios a través de resultados del SPSS.21.0

A partir de los resultados del cuestionario se determinó desarrollar la propuesta de un sistema de indicadores para medir el comportamiento del consumidor para la sostenibilidad económica del destino Sucre San Vicente a través de los siguientes pasos, los cuales se describen a continuación:

Paso 1:Tiene como objetivo el marco teórico de la investigación, e identificar los indicadores más comunes que se utilizan en el marketing relacional y comportamiento del consumidor. Para esto se realizó: la revisión bibliográfica y método Delphi. 
Para esto se seleccionó como expertos, a profesionales docentes de las facultades de marketing y turismo con experiencia en estudios de comportamiento al consumidor y conocimientos probados por sus resultados investigativos que aporten al desarrollo sostenible del destino turístico Sucre San Vicente. Se necesitará indicar si los indicadores que se detallen a continuación serian útiles, en caso afirmativo será marcado la casilla SI, caso contrario se marcara la casilla NO.

Los expertos seleccionados son investigadores del área que han sido recomendados por investigadores de nuestras redes universitarias. Los indicadores se muestran en la tabla 7.

Tabla 4. Indicadores seleccionados

\begin{tabular}{l}
\hline Indicadores \\
\hline Calidad del servicio \\
\hline Realizar ventas cruzadas de manera más eficiente \\
\hline Frecuencia de compra \\
\hline Necesidades, deseos y demanda de los consumidores \\
\hline Aumento sustancial de las compras \\
\hline Desarrollo de la rentabilidad \\
\hline Integración del marketing mix y marketing digital \\
\hline Crecimiento significativo de las transacciones \\
\hline Satisfacción continua de los clientes (producto servicio al cliente) \\
\hline Cumplimiento de los objetivos organizacionales \\
\hline Gestión adecuada de quejas y sugerencias \\
\hline Utilización del producto \\
\hline Expectativas reales del producto o servicio \\
\hline Percepción de la marca y envase \\
\hline Instalaciones de la empresa \\
\hline Ofertas personalizadas a clientes (CRM) \\
\hline Herramientas de comunicación empresarial \\
\hline Calidad percibida por los clientes \\
\hline Imagen de los vendedores \\
\hline Preferencias de marcas \\
\hline
\end{tabular}

Fuente: Elaborado con datos propios a través de criterios de expertos. 
Paso 2: Se analizan el tipo de consumidor que sobresalen en el marketing relacional y que se vinculan con el entorno de las empresas hoteleras.

$\mathrm{Al}$ realizar la investigación se pudo contactar que no existe un sistema de indicadores para evaluar el comportamiento del consumidor para la sostenibilidad económica del destino que permita medir a cabalidad con sus objetivos propuestos para el posicionamiento de su servicio de alojamiento.

Paso 3: Determinación de los indicadores para evaluar el comportamiento del consumidor en correspondencia al objeto estudio de la investigación. El objetivo que tiene este paso es definir el sistema de indicadores que evalúe el comportamiento del consumidor.

Partiendo de una lista inicial de 20 indicadores se aplica el método Delphi, para ellos se utilizó a 16 expertos, y se realizó la selección de expertos aplicando la metodología de Ońate Ramos cuyo procedimiento consta de tres fases.

De los 16 expertos a evaluar 5 son $\mathrm{PhD}$ de nacionalidad cubana, con más de 10 años en marketing relacional, 4 son brasileños magister, 2 son colombianos con maestría en comportamiento del consumidor, 5 son ecuatorianos con 4to nivel de ingeniería en marketing.

Para la selección de expertos se aplica la metodología de expertos de Ońate Ramos, que evalúa el coeficiente de experticidad a partir del conocimiento de científicos y concordancias. Los expertos a evaluar, todos poseen más de 10 ańos de experiencia en marketing relacional y comportamiento del consumidor.

\section{Método Delphi}

El primer estudio de Delphi fue realizado en 1950 por la Rand Corporation para la fuerza aérea de Estados Unidos, y se le dio el nombre de Proyecto Delphi. Su objetivo era la aplicación de la opinión de expertos a la selección de un sistema industrial norteamericano óptimo y la estimación del número de bombas requeridas para reducir la producción de municiones hasta un cierto monto. Es un método de estructuración de un proceso de comunicación grupal que es efectivo a la hora de permitir a un grupo de individuos, como un todo, tratar un problema complejo. Linstone, H \& Turoff, M (1975) 
El instrumento que se somete a validación por el panel de expertos es un sistema constituido por 20 indicadores, donde se evaluaron la relevancia, la pertinencia y la coherencia del Sistema de Indicadores propuesto.

La validación del sistema se realizó mediante el método Delphi para la consulta a expertos, según el grado de conocimiento de los mismos, lo cual le dio un carácter participativo al proceso, teniendo en cuenta que estos indicadores son aplicables al desarrollo local.

Procedimiento: Siguiendo los criterios de un grupo de autores que han aplicado el método Delphi en sus investigaciones Oñate et al 1998 muestran cómo se estableció la secuencia metodológica a seguir, compuesta de tres fases fundamentales: preliminar, exploratoria y final.

Fase preliminar: En esta primera fase se conformó el grupo coordinador que asumió la responsabilidad de seleccionar el grupo de expertos a partir de su nivel de conocimiento y competencia. Entre sus funciones estuvo interpretar los resultados parciales y finales de la investigación y supervisar la marcha correcta de la misma, para realizar ajustes y correcciones. El procedimiento para la conformación de los dos grupos que intervinieron en la investigación se explica en el apartado correspondiente a la muestra.

Fase exploratoria: Se confeccionó la primera versión del cuestionario y se sometió, en una segunda ronda, a la validación por el grupo de expertos seleccionados con la intención de recabar los criterios cuantitativos y cualitativos más estables.

El envío y la recepción del cuestionario se realizó por correo electrónico en archivo adjunto, el cual estaba conformado por una primera página con una breve introducción al tema de investigación, la explicación clara del objetivo de la investigación, el método que se utiliza, la fase en que se encontraba el proceso de investigación, las instrucciones para cumplimentar el cuestionario, seguido del correspondiente instrumento para la validación. Este último consta de una escala de respuesta con dos alternativas, tomando en cuenta como plazo máximo dado para responder de 20 días. Se le asignó un número a cada experto para garantizar el anonimato.

Los resultados de la selección de expertos aplicando la metodología de Ońate Ramos fueron analizados desde el punto de vista cuantitativo 
tomando en consideración el coeficiente de conocimiento, coeficiente de argumentación, coeficiente de competencia de los expertos para después arrojar su validación.

Fase Final: En esta última fase se solicita la valoración por parte de los expertos en cuanto a la relevancia, la pertinencia y la coherencia del sistema de indicadores para prever, medir y evaluar el comportamiento del consumidor en correspondencia al marketing relacional de esta investigación, resumiendo los resultados que los expertos propusieron en definitiva un sistema de indicadores para su posterior aplicación en el proceso de investigación.

Tabla 5. Resultados finales por expertos

Resultado del procesamiento para la determinación del coeficiente de competencia propuesto por ońate ramos

\begin{tabular}{ccccc}
\hline Expertos & $\begin{array}{c}\text { Coeficiente de } \\
\text { Conocimiento } \\
(\mathrm{kc})\end{array}$ & $\begin{array}{c}\text { Coeficiente de } \\
\text { Argumentación } \\
(\mathrm{Ka})\end{array}$ & $\begin{array}{c}\text { Coeficiente de } \\
\text { competencia }(\mathrm{K})\end{array}$ & Validación \\
\hline 1 & 0,9 & 1 & 0,95 & Alto \\
\hline 2 & 0,9 & 1 & 0,95 & Alto \\
\hline 3 & 0,1 & 0,9 & 0,95 & Alto \\
\hline 4 & 0,8 & 0,9 & 0,85 & Alto \\
\hline 5 & 0,8 & 1 & 0,9 & Alto \\
\hline 6 & 0,9 & 1 & 0,95 & Alto \\
\hline 7 & 0,8 & 0,8 & 0,8 & Alto \\
\hline 8 & 1 & 1 & 1 & Alto \\
\hline 9 & 0,8 & 0,9 & 0,85 & Alto \\
\hline 10 & 0,8 & 0,9 & 0,85 & Alto \\
\hline 11 & 0,8 & 0,8 & 0,8 & Alto \\
\hline 12 & 0,8 & 0,9 & 0,85 & Alto \\
\hline 13 & 0,8 & 0,9 & 0,85 & Alto \\
\hline 14 & 0,8 & 0,9 & 0,85 & Alto \\
\hline 15 & 0,8 & 1 & 0,9 & Alto \\
\hline 16 & 0,8 & 1 & 0,9 & Alto \\
\hline
\end{tabular}

Fuente. Elaborado con datos propios a través resultados de criterio de expertos 
Leyenda:

$\mathrm{Kc}=$ Coeficiente de conocimiento

$\mathrm{Ka}=$ Coeficiente de argumentación

$\mathrm{K}=$ Coeficiente de competencia de los expertos

Como se observa el coeficiente de experticia de los 16 profesionales evaluados se califica como alto, atendiendo a su nivel de conocimiento y argumentación. Se determina un coeficiente de concordancia Delphi de 0,85 , ósea que aquel indicador que tenga más de 4 votos negativos según la experticia de los evaluadores será eliminado. Al calcular el coeficiente de concordancia real y coeficiente prefijo se eliminan 10 indicadores quedando como resultado 10 indicadores siendo estos los siguientes:

Para esto se utiliza la fórmula de coeficiente de concordancia de resultados, siendo así su fórmula:

$$
\mathrm{CCR}=1-\mathrm{VN} / \mathrm{VT}
$$

Tabla 6. Indicadores de Comportamiento del Consumidor

\begin{tabular}{|c|c|c|}
\hline $\mathbf{N}^{\circ}$ & Indicadores & $\begin{array}{l}\text { CCR. Coeficientes } \\
\text { de concordancia } \\
\text { de resultados }\end{array}$ \\
\hline 1. & Calidad del Servicio & 1 \\
\hline 2. & Calidad percibida de los clientes & 0,96 \\
\hline 3. & Frecuencia de compra & 0,92 \\
\hline 4. & Integridad de marketing mix y marketing digital & 0,94 \\
\hline 5. & Expectativas reales de producto o servicio & 1 \\
\hline 6. & Necesidades, deseo y demanda de los consumidores & 1 \\
\hline 7. & Ofertas personalizadas a los clientes (CRM) & 0,88 \\
\hline 8. & $\begin{array}{l}\text { Satisfacción continua de los clientes (productos y } \\
\text { servicios al cliente) }\end{array}$ & 0,92 \\
\hline 9. & Gestión adecuada de quejas y sugerencias & 0,88 \\
\hline 10. & Herramientas de comunicación empresarial & 1 \\
\hline
\end{tabular}

Fuente. Elaborado con datos propios a través de la aplicación método Delphi 
Como se puede observar cuatro de los 10 indicadores fueron los mejores evaluados por los expertos, donde 4 fueron evaluado por encima del $90 \%$ y dos con un $88 \%$, indicando que los resultados obtenidos fueron resultados muy buenos.

\section{Conclusiones}

La bibliografía revisada permitió definir los indicadores a medir para poder determinar el coeficiente de competencia según Ońate Ramos donde la validación por experto estableciendo la secuencia metodológica a seguir, compuesta de tres fases fundamentales: preliminar, exploratoria y final para determinar los indicadores para medir comportamiento de consumidores para definir sostenibilidad económica del destino Sucre-San Vicente.

El cuestionario aplicado arrojo resultados favorables donde la variedad de las ofertas turísticas que brinda el hotel y a los servicios del restaurante está acorde a las expectativas representando un $42,1 \%$ que reconoce que es lo esperado y un $28,4 \%$ sugirió que es mucho más de lo esperado, pero la ubicación de los centros recreativos $26,3 \%$ manifiestan que era lo que ellos esperaban, mientras que el $43,2 \%$ de los encuestados opinaron que la seguridad turística en este hotel es igual a lo esperado, donde la fiabilidad del instrumento fue de 0.87

Los resultados de la selección de expertos aplicando la metodología de Oñate Ramos en consideración al coeficiente de conocimiento, coeficiente de argumentación, coeficiente de competencia de los expertos arrojaron resultados satisfactorios dado en sus votaciones impacto alto, donde cuatros de los 10 indicadores fueron los mejores evaluados por los expertos, donde 4 fueron evaluado por encima del $90 \%$ y dos con un $88 \%$, indicando que los resultados obtenidos fueron resultados muy buenos.

\section{Bibliografía}

Beltrán, M. Á., \& Parra, M. C. (2017). Perfiles turísticos en función de las motivaciones para viajar. Cuadernos de Turismo, 39: 41-65.

Carbache, M., Carbache, W., Macas, C., \& Orden, M. (2018). Motivaciones, Valoración y Satisfacción del Turista en un destino de Sol y Playa de Ecuador. ESPACIOS, 39(13):4. 
Carvajal, G. V., Valls, W., Lemoine, F. Á., \& Alcívar, V. E. (2017). Gestión por procesos: Un principio de la gestión de calidad. Manta, Manabí, Ecuador: Mar Abierto

Conde, E. M., Amaya, C. M., \& González, E. (2013). Factores que influyen en el comportamiento del consumidor turista: el caso de Manzanillo, México. Teoría y Praxis, 14: 109-139

Cruz, M., \& Rúa, J. A. (2018). Surgimiento y desarrollo del método Delphi: una perspectiva cienciométrica. Biblios, 71:91-107. DOI 10.5195/biblios.2018.470

Escalante J.L. (2016). Marketing Link. Obtenido de Los principales factores que influyen en la conducta del consumidor: http:// marketinglink.up.edu.pe/los-principales-factores-influyen-la-conducta-del-consumidor/

Fernández, J. M. (2012). Oportunidad y conveniencia de aplicar la prospectiva al sector turismo. Estudios Turísticos, 192: 7-33

Flores, S., Mora, L., \& Rivero, J. Á. (2019). El método Delphi aplicado al diseño de un modelo de financiación de transporte urbano. Economía, Sociedad y Territorio, 19(61):575-600

Gallardo, G. (2013). Evaluación del potencial turístico de las playas del departamento del Atlántico - Colombia, desde la perspectiva ambiental. Dimensión empresarial, 11(2): 62-69

García, N., Carreño, Á. L., \& Doumet, N. (2020). Validación del modelo de gestión sostenible para el desarrollo turístico en vinculación universidad - comunidades manabitas. Ecuador. Investigación \& Negocio,13(21):37-51 DOI: https://doi.org/10.38147/inv\&neg. v13i21.82

Harold A. Linstone y Murray Turoff. (1975). The Delphi Method: Techniques and Applications. San Francisco: Addison-Wesley

Kotler P \& Armstrong G. (2007-2008). Marketing: Versión para Latinoamérica. Decimoprimera edición. México: Pearson Educación de México, S. A. de C. V

Lemoine, F. Á., Montesdeoca, M. G., Villacís, L. M., \& Hernández, N. R. (2020). El comportamiento del consumidor en la gestión comercial de destinos turísticos Sucre-San Vicente. Un acercamiento desde las ciencias sociales, ecuador. 3C Empresa. Investigación y pensamiento crítico, 9 (1): 17-39. DOI: 10.17993/3ctecno. 2017. v6n4e24.64-73 
Ministerio de Turismo del Ecuador. (2017). Gobierno Nacional presentó la Política Nacional de Turismo, que convertirá al país en potencia turística. Obtenido de https://www.turismo.gob.ec/gobierno-nacional-presento-la-politica-nacional-de-turismo-que-convertira-al-pais-en-potencia-turistica/

MINTUR. (2016). Boletín de Estadísticas Turísticas. Ecuador, Quito: Ministerio de Turismo

Morales, J. R., Arévalo, D. X., Padilla, C. P., \& Bustamante, M. A. (2018). Nivel de Satisfacción e Intención de Repetir la Visita Turística. El Caso del Cantón Playas, en Ecuador. Información Tecnológica, 29(1): 181-192.http://dx.doi.org/10.4067/S071807642018000100181,

Narváez, M., \& Fernández, G. (2010). El turismo desde la perspectiva de la demanda. Lugar de estudio: península de Paraguaná-Venezuela. Turismo y demanda turística:175-183

OMT. (2018). Panorama Organización Mundial del Turismo. Obtenido de mkt.unwto.org

Ońate N, Ramos, L \& Díaz, A. (1988). Utilización del Método Delphi en la pronosticación: Una experiencia inicial. Cuba: Economía Planificada

PEGEASOSTENIBLE. (2012). PEGEASOSTENIBLE. Obtenido de Federación Plurinacional de Turismo Comunitario del Ecuador: https://pangeasostenible.org/2011/12/federacion-plurinacional-de-turismo-comunitario-del-ecuador/

Picazo, P., \& Moreno, S. (2013). Difusión de la investigación científica en turismo. El caso de México. El Periplo Sustentable, 24: 7-40 https://doi.org/10.36677/elperiplo.v0i39

Pulido, J. I., \& Ruiz, A. (2017). Propuesta para una agenda de investigación de los impactos del turismo en los destinos patrimonio de la humanidad. Boletín de la Asociación de Geógrafos Españoles, 3:389-411

Ruiz, F. I. (2016). Desarrollo de una metodología para determinar el potencial del turismo rural en Oasis sudcalifornianos de México. Revista Global de Negocios, 4(8): 23-34 
Sánchez, E. (2015). Transporte. Guía Ecuatoriana de Transporte y Turismo. Obtenido de https://transport.ec/opinion-2/ha-evolucionado-la-industria-hotelera-y-turistica-en-el-ecuador/

SECTUR. (2014). Evaluación de desempeño de los destinos turísticos en el marco de los Convenios de Coordinación en materia de Reasignación de Recursos (CCRR). Obtenido de www.cestur. sectur.gob.mx

Solomon, M. (2008). Comportamiento del consumidor. Séptima edición. México: Pearson Educación

Solsona, J. (2014). Análisis prospectivo del turismo rural: el caso de la comunitat valenciana. Cuadernos de Turismo, 34: 313-334

Torres, L. (2016). Factores que inciden en la evaluación del desarrollo sostenible de destinos turísticos. El caso de Cuba. Retos Turísticos, $15(1)$

Vargas, Alfonso; Vaca, Rosa; García, Elena. (2014). Sostenibilidad De La Empresa Hotelera: Indicadores para su Medición (Responsabilidad Medioambiental). Obtenido de https:/www.uhu.es/ alfonso_vargas/archivos/UEMA\%202005.pdf 
